\title{
Evaluation of a cryptococcal antigen lateral flow assay test for rapid detection of cryptococcal infection in HIV-negative patients in Ibadan, Nigeria
}

\author{
${ }^{* 1,2,4}$ Fayemiwo, S. A., ${ }^{1,2}$ Makanjuola, O. B., ${ }^{1}$ Nwaokenye, J., and ${ }^{3}$ Owolabi, M. O. \\ ${ }^{1}$ Department of Medical Microbiology and Parasitology, University College Hospital, Ibadan, Nigeria \\ ${ }^{2}$ Department of Medical Microbiology and Parasitology, College of Medicine, University of Ibadan, Ibadan, Nigeria \\ ${ }^{3}$ Department of Medicine, College of Medicine, University of Ibadan, Ibadan, Nigeria \\ ${ }^{4}$ Division of Infection, Immunity and Respiratory Medicine, School of Biological Sciences, \\ University of Manchester, Manchester, M13 9PL, UK \\ *Correspondence to: dayteet@yahoo.com
}

\begin{abstract}
:
Background: A number of studies have been conducted in Nigeria on the prevalence of cryptococcal infections mostly on HIV-infected patients using culture, India ink and/or latex agglutination tests. These tests are either laborious, time-consuming and expensive or have low sensitivity, thus limiting their use. Cryptococcal antigen lateral flow assays (LFA) were introduced in the last decade as rapid user-friendly tests for diagnosis. In this study, we sought to determine the diagnostic accuracy of an LFA kit for the detection of cryptococcal antigen in the serum of HIV-negative patients with or without cerebrovascular accident (CVA) or stroke in University College Hospital, Ibadan, Nigeria.

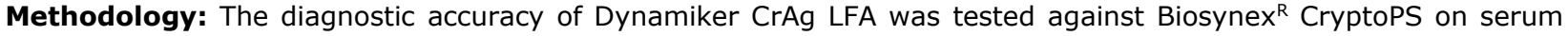
samples of 100 HIV-negative patients with and without stroke. Samples were tested and results interpreted in accordance with the manufacturer's instructions. The sensitivity, specificity, positive predictive value (PPV), negative predictive value (NPV), and positive and negative likelihood ratios of the Dynamiker CrAg LFA were calculated by comparing with the Biosynex ${ }^{R}$ CryptoPS as 'gold standard'.

Results: Overall, a total of 98 valid patient sample results were analysed; 17 samples (17.3\%) were positive with Dynamiker CrAg LFA cryptococcal antigen and 16 samples (16.3\%) were positive with Biosynex ${ }^{R}$ CryptoPS. The sensitivity, specificity, PPV and NPV of Dynamiker CrAg LFA compared to the Biosynex ${ }^{\mathrm{R}}$ CryptoPS were $100 \%, 98.8 \%$, $94.1 \%$ and $100 \%$ respectively, while the positive and negative likelihood ratios were 82 and 0 respectively. Conclusion: In comparison to the Biosynex ${ }^{R}$ CryptoPS, the Dynamiker CrAg LFA is a highly sensitive and specific test for the detection of cryptococcal antigen in serum. The test kit should be considered as a screening device for cryptococcal infection both in outreach and clinical settings, especially in antiretroviral therapy (ART) centres.
\end{abstract}

Keywords: Cryptococcus; evaluation; lateral flow assay; HIV-negative; stroke

Received April 23, 2020; Revised July 3, 2020; Accepted July 4, 2020

Copyright 2021 AJCEM Open Access. This article is licensed and distributed under the terms of the Creative Commons Attrition 4.0 International License <a rel="license" href="http://creativecommons.org/licenses/by/4.0/", which permits unrestricted use, distribution and reproduction in any medium, provided credit is given to the original author(s) and the source. Editor-in-Chief: Prof. S. S. Taiwo

\section{Évaluation d'un test de test d'écoulement latéral de l'antigène cryptococcique pour la détection rapide d'une infection cryptococcique chez des patients séronégatifs à Ibadan, Nigéria}

\author{
${ }^{* 1,2,4}$ Fayemiwo, S. A., ${ }^{1,2}$ Makanjuola, O. B., ${ }^{1}$ Nwaokenye, J., et ${ }^{3}$ Owolabi, M. O.
}

${ }^{1}$ Département de microbiologie médicale et de parasitologie, University College Hospital, Ibadan, Nigéria ${ }^{2}$ Département de microbiologie médicale et de parasitologie, Collège de médecine,

Université d'Ibadan, Ibadan, Nigéria

${ }^{3}$ Département de médecine, Collège de médecine, Université d'Ibadan, Ibadan, Nigéria 


\author{
${ }^{4}$ Division de l'infection, de l'immunité et de la médecine respiratoire, École des sciences biologiques, \\ Université de Manchester, Manchester, M13 9PL, Royaume-Uni \\ *Correspondance à: dayteet@yahoo.com
}

\begin{abstract}
Abstrait:
Contexte: Un certain nombre d'études ont été menées au Nigéria sur la prévalence des infections à cryptocoques principalement chez les patients infectés par le VIH en utilisant des tests d'agglutination en culture, à l'encre de Chine et / ou au latex. Ces tests sont soit laborieux, longs et coûteux ou ont une faible sensibilité, limitant ainsi leur utilisation. Les tests d'écoulement latéral de l'antigène cryptococcique (LFA) ont été introduits au cours de la dernière décennie en tant que tests conviviaux rapides pour le diagnostic. Dans cette étude, nous avons cherché à déterminer la précision diagnostique d'un kit LFA pour la détection de l'antigène cryptococcique dans le sérum de patients séronégatifs avec ou sans accident vasculaire cérébral (AVC) ou accident vasculaire cérébral à University College Hospital, Ibadan, Nigeria.

Méthodologie: La précision diagnostique de Dynamiker CrAg LFA a été testée contre Biosynex ${ }^{R}$ CryptoPS sur des échantillons de sérum de 100 patients séronégatifs avec et sans accident vasculaire cérébral. Les échantillons ont été testés et les résultats interprétés conformément aux instructions du fabricant. La sensibilité, la spécificité, la valeur prédictive positive (PPV), la valeur prédictive négative (NPV) et les rapports de vraisemblance positifs et négatifs du Dynamiker CrAg LFA ont été calculés en les comparant avec le Biosynex ${ }^{\mathrm{R}}$ CryptoPS comme 'gold standard'. Résultats: Au total, 98 résultats d'échantillons de patients valides ont été analysés; 17 échantillons (17,3\%) étaient positifs avec l'antigène cryptococcique Dynamiker CrAg LFA et 16 échantillons $(16,3 \%)$ étaient positifs avec Biosynex ${ }^{R}$ CryptoPS. La sensibilité, la spécificité, le PPV et le NPV de Dynamiker LFA par rapport au Biosynex ${ }^{R}$ CryptoPS étaient respectivement de $100 \%, 98,8 \%, 94,1 \%$ et $100 \%$, tandis que les rapports de vraisemblance positifs et négatifs étaient respectivement de 82 et 0 .

Conclusion: Par rapport au Biosynex ${ }^{R}$ CryptoPS, le Dynamiker CrAg LFA est un test hautement sensible et spécifique pour la détection de l'antigène cryptococcique dans le sérum. Le kit de test doit être considéré comme un dispositif de dépistage de l'infection cryptococcique à la fois dans les milieux de proximité et cliniques, en particulier dans les centres de thérapie antirétrovirale (ART).
\end{abstract}

Mots-clés: Cryptococcus; évaluation; essai d'écoulement latéral; Séronégatif; accident vasculaire cérébral

\section{Introduction:}

Cryptococcosis is a systemic fungal infection caused by Cryptococcus neoformans and Cryptococcus gatti complex (1). These fungi are considered potential pathogenic species and infect both human and animal hosts. While it is indeed true that $C$. neoformans infections are commoner among the immunocompromised, $C$. gatti, on the other hand is more frequently implicated in fungal infections among immunocompetent hosts. The commonest infections they cause in humans include meningitis, pneumonia and disseminated cryptococcosis, mostly in immunosuppressed hosts. Sporadic cases of these infections are reported all over the world. However, in recent times, the AIDS pandemic has been the significant factor for increased incidence of this disease. Cryptococcal meningitis is one of the common causes of unconsciousness and adult meningitis in Africa, (2) resulting in about $20-25 \%$ of AIDS-related deaths $(3,4)$.

Although, the major risk factors for cerebrovascular accident (CVA) or stroke in the Nigerian population include hypertension, alcohol consumption, sedentary lifestyle, and impaired glucose tolerance, these factors do not entirely explain the occurrence of stroke. Meanwhile, patients with uncontrolled hyper- tension who usually suffer stroke present at the hospital in coma in the Nigerian setting. Coma, being a common presentation of both cryptococcal meningitis and stroke, may thus present a diagnostic challenge, necessitating a rapid screening to exclude cryptococcal infection (5). Laboratory diagnosis of cryptococcal infections have traditionally been carried out by CSF culture for isolation of the pathogen, India ink test and/or cryptococcal antigen latex agglutination tests. However, these tests are either laborious, time-consuming, expensive or have low sensitivity, thus limiting their clinical use. For instance, the 'gold standard' test for the diagnosis of cryptococcal meningitis is CSF culture but diagnosis by culture can take up to 1 or 2 weeks for definitive results. India ink test, though quick, has a low sensitivity resulting in missed infections (6). Thus, other test methods have been devised to expedite diagnosis and treatment, thus improving clinical outcome.

The cryptococcal antigen lateral flow assay (LFA) tests were introduced in the last decade of this century as rapid user-friendly tests for diagnosis of Cryptococcus infections. The use of these assays has simplified the presumptive diagnosis of cryptococcal infection as point-of-care test using serum/plasma, whole blood and cerebrospinal fluid (CSF). The LFA have been reported to demonstrate high accuracy 
in these body fluids for the diagnosis of cryptococcosis in patients at risk of infection (7).

The first of these kits was manufactured by IMMY and has been validated and licensed for use in many countries. Other LFA kits such as the Biosynex ${ }^{R}$ CryptoPS have been found to also perform well $(6,8,9)$, with comparable diagnostic accuracy to the IMMY CrAg kit that is considered the 'gold standard' LFA test on serum samples (8). Furthermore, other newer test kits have been manufactured and are in need of evaluation. We therefore evaluated the diagnostic accuracy of the Dynamiker CrAg LFA test against Biosynex ${ }^{R}$ CryptoPS using sera of HIVnegative patients with and without stroke in Ibadan, southwest Nigeria.

\section{Materials and method:}

\section{Study setting}

The study was conducted in Ibadan, southwestern Nigeria as part of the Stroke Investigative Research and Education Network (SIREN) project, a multicentre and multidisciplinary study on stroke in sub-Saharan Africa.

\section{Study design, subjects and sample collection}

This was a comparative evaluation study of the diagnostic accuracy of the cryptococcal CrAg LFA (Dynamiker Biotechnology, Tianjin Co., Ltd,) against Biosynex ${ }^{R}$ CryptoPS kits on sera of 100 HIV-negative adult patients aged $\geq 18$ years with and without stroke, randomly selected from the SIREN project.

Approximately $5 \mathrm{ml}$ of venous blood samples were collected from each patient. Blood samples were allowed to clot and serum was separated by centrifugation and stored at $-80^{\circ} \mathrm{C}$ until use. The study was approved by the University of Ibadan/University College Hospital Ethics Review Committee.

\section{Sample testing by the lateral flow assay kits}

The serum samples were allowed to thaw for at least one hour at room temperature, and then tested against the Dynamiker CrAg (LOT 17040) and Biosynex ${ }^{R}$ CryptoPS kits strictly in accordance with the manufacturer's instructions.

\section{Principle of the lateral flow device}

Both test kits use the lateral flow technique and double antibody sandwich format. In the kit, the test cassette consists of Cryptococcus antibodies bound to gold particles and coated on fibre glass. The cassette has a test line ( $T$ ) and a control line (C) on nitrocellulose membrane which are coated with Cryptococcus and goat anti-mouse antibodies respectively. The cryptococcal antigens if present in the test samples form antigen-antibody complexes with the gold-conjugated antibodies and migrate further along the nitrocellulose membrane. The antibodies immobilized on the test line $(T)$ interact with the above complexes causing a visible red line. The wicking of samples causes free gold-conjugated antibodies to reach the control line $(C)$ to form red colour. The absence of cryptococcal antigens in negative samples only cause a colour change in the control line (C), which is essentially a quality control measure to ensure the test is visually validated to be functioning properly.

\section{Procedural steps}

Following strictly the instructions of the manufacturer for the Dynamiker CrAg LFA, the test kit was removed from the pouch and placed on a flat surface. $80 \mu \mathrm{L}$ of the serum sample was gently dispensed onto the sample pad and result read within 15-20 minutes, and interpreted as positive, negative or invalid. Where interpretation was difficult due to high viscosity of serum sample, such sample was re-centrifuged and the test repeated.

For the Biosynex ${ }^{R}$ CryptoPS, the test kit was similarly removed from the pouch and placed on a flat, horizontal surface. $20 \mu \mathrm{L}$ of the serum sample was dispensed into the well, followed by 3 drops of the diluent. Result was read and interpreted as positive, negative or invalid in accordance with the manufacturer's instructions.

\section{Data analysis}

The sensitivity, specificity, positive and negative predictive values of the Dynamiker $\mathrm{CrAg}$ test were calculated in reference to the Biosynex $^{R}$ CryptoPS using the formula; sensitivity $=T P / T P+F N \times(100)$, specificity $=T N / T N+F P$ $x(100), P P V=T P / T P+F P x(100)$ and NPV $=T N /$ $\mathrm{TN}+\mathrm{FN} \times(100)$, where $\mathrm{TP}=$ True Positive, $\mathrm{TN}=$ True Negative, $F P=$ False Positive and $F N=$ False Negative. The positive likelihood ratio (PLR) and negative likelihood ratio (NLR) were also computed as follows; NLR $=(1$-sensitivity $) /$ specificity and PLR=sensitivity/(1-specificity).

\section{Results:}

A total of 100 serum samples from 100 HIV-negative patients with or without stroke were tested by both the Dynamiker CrAg LFA and Biosynex ${ }^{R}$ CryptoPS kits. After the initial test, 12 samples produced invalid results for the two kits but on repeat testing, only 2 samples remained invalid. A total of 98 sample results were therefore analysed. Seventeen samples 
(17.3\%) were positive for Dynamiker CrAg LFA while $16(16.3 \%)$ were positive for Biosynex ${ }^{R}$ CryptoPS assay (Table 1). The sensitivity, specificity, PPV and NPV of Dynamiker CrAg LFA compared to the 'gold standard' Biosynex CryptoPS were $100 \%, 98.8 \%, 94.1 \%$ and $100 \%$ respectively. The positive and negative likelihood ratios were respectively 82 and 0 (Table 2 ).

Table 1: Cryptococcal antigen testing using Dynamiker CrAg LFA and Biosynex ${ }^{R}$ CryptoPS assay kits

\begin{tabular}{llll}
\hline Diagnostic kit & \multicolumn{2}{l}{ BiosynexR CryptoPS } \\
\cline { 2 - 4 } & Positive & Negative & Total \\
Dynamiker CrAg LFA & & & \\
Positive & 16 & 1 & 17 \\
Negative & 0 & 81 & 81 \\
Total & 16 & 82 & 98 \\
\hline
\end{tabular}

Sensitivity of Dynamiker CRAG LFA=16/16 (100\%); Specificity $=81 / 82(98.8 \%)$; Negative Predictive Value (NPV) $=81 / 81(100 \%)$; Positive Predictive Value $(\mathrm{PPV})=16 / 17(94.1 \%)$; Negative Likelihood Ratio $=0.0$; Positive Likelihood Ratio $=$ 82.0; Disease Prevalence $=16.3 \%$.

Table 2: Summary of the diagnostic performance of Dynamiker CrAg LFA compared to the 'gold standard'

\begin{tabular}{lllll}
\hline Characteristics & Sensitivity & Specificity & NPV & PPV \\
\hline Value (\%) & 100 & 98.8 & 100 & 94.1 \\
& & & & \\
$95 \%$ CI & $79.4-100$ & $93.4-99.9$ & $92.4-$ & $69.5-$ \\
& & & 99.8 & 99.1
\end{tabular}

$\overline{N P V}=$ Negative Predictive Value; PPV=Positive Predictive Value; CI=Confidence Interval

\section{Discussion:}

In this study, the Dynamiker CrAg LFA was highly sensitive and specific in detecting cryptococcal antigen in the serum of HIVnegative patients with and without stroke when compared to Biosynex ${ }^{R}$ CryptoPS. The negative predictive value was $100 \%$ while the positive predictive value was $94.1 \%$. These results imply that the probability of cryptococcal disease not being present when the test is negative is $100 \%$, while the probability that the disease is present when the test is positive is $94.1 \%$. The positive likelihood ratio was greater than 20 while the negative likelihood ratio was less than 0.1 , which proved that ill people with cryptococcal infection are more likely to have abnormal test results compared to healthy people.

The Dynamiker CrAg test was simple to use and rapid in detecting cryptococcal antigen in the serum samples of the patients. The test requires minimal training or equipment and therefore should be considered as a screening device both in outreach and clinical setting especially in ART centres.
Our study was limited by the inability to perform this evaluation with the more preferred IMMY CrAg test that is considered to be the LFA 'gold standard' test for detecting cryptococcus infection. This limitation was however compensated for by the use of the Biosynex ${ }^{R}$ CryptoPS kit which has been reported to have comparable diagnostic accuracy with IMMY CrAg test and is CE-marked for use in Europe (8).

\section{Conflict of interest:}

Authors declare no competing interest

\section{Acknowledgements:}

The authors appreciate the staff of the SIREN study team of College of Medicine, University of Ibadan. The authors also acknowledge with thanks the resident doctors, nurses, laboratory scientists and administrative staff of the UCH Medical Mycology Research Group (MRG) for their support during the procedure and analysis.

\section{References:}

1. Viviani, M. A., Cogliati, M., Esposto, M. C., et al. Molecular analysis of 311 Cryptococcus neoformans isolates from a 30-month ECMM survey of cryptococcosis in Europe. FEMS Yeast Research. 2006; 6 (4): 614-619.

2. Boulware, D. R., Rolfes, M. A., Rajasingham, R., et al. Multisite validation of cryptococcal antigen lateral flow assay and quantification by laser thermal contrast. Emerg Infect Dis. 2014; 20 (1): 45-53.

3. Oladele, R. O., and Denning, D. W. Burden of serious fungal infection in Nigeria. West Afr J Med. 2014; 33 (2): 107-114.

4. Kwizera, R., Nguna, J., Kiragga, A., et al. Performance of cryptococcal antigen lateral flow assay using saliva in Ugandans with CD4 < 100. PLoS One. 2014; 9 (7): e103156.

5. Lai, C. H., Lin, G. Y., Lee, J. T., et al. A Rare Case of Cryptococcus Meningoencephalitis Presenting with Acute Brainstem Infarction in an Immuno-competent Host. Neuropsychiatry. 2017; 7(6): 812- 815.

6. Rajasingham, R., Wake, R. M., Beyene, T., Katende, A., Letang, E., and Boulware, D. R. Cryptococcal Meningitis Diagnostics and Screening in the Era of Point-of-Care Laboratory Testing. J Clin Microbiol. 2019; 57(1): e01238-18

7. Huang, H. R., Fan, L. C., Rajbanshi, B., and Xu, J. F. Evaluation of a new cryptococcal antigen lateral flow immunoassay in serum, cerebrospinal fluid and urine for the diagnosis of cryptococcosis: a meta-analysis and systematic review. PLoS One. 2015; 10 (5): e0127117.

8. Temfack, E., Kouanfack, C., Mossiang, L., et al. Cryptococcal Antigen Screening in Asymptomatic HIVInfected Antiretroviral Naive Patients in Cameroon and Evaluation of the New Semi-Quantitative Biosynex ${ }^{R}$ CryptoPS Test. Front Microbiol. 2018; 9: 409

9. Caceres, D. H., Zuluaga, A., Tabares, A. M., Chiller, T., Gonzalez, A., and Gomez, B. L. Evaluation of a Cryptococcal antigen Lateral Flow Assay in serum and cerebrospinal fluid for rapid diagnosis of cryptococcosis in Colombia. Rev Inst Med Trop Sao Paulo. 2017; 59: e76. 